\title{
ENSEÑAR A PENSAR EN EL AULA UNIVERSITARIA. UNA EXPERIENCIA EN LAS AULAS DE MAESTRO DE LA UNIVERSIDAD DE LA RIOJA
}

\author{
Alicia Pérez de Albéniz Iturriaga \\ María Teresa Pascual Sufrate \\ Universidad de La Rioja
}

RESUMEN: La labor de un maestro requiere no sólo un conocimiento exhaustivo sobre las posibles necesidades especiales de sus alumnos sino que además debe ser crítico y creativo en las intervenciones que realiza con estos alumnos. En el contexto de la asignatura Bases Psicológicas de la Educación Especial de la Diplomatura de Maestro de la Universidad de La Rioja, se llevó a cabo una experiencia innovadora con el objetivo de ayudar a los alumnos a desarrollar estas competencias. En concreto, el presente trabajo tenía como objetivo la activación de un pensamiento crítico sobre las ideas previas que tienen los alumnos acerca de las necesidades educativas especiales y sobre la información que se proporciona en el aula. Asimismo, se pretendía desarroIlar un pensamiento creativo sobre posibles actuaciones que pueden realizar con sus propios alumnos ante sus necesidades. Se trabajaron dos temas con esta metodología (altas capacidades y trastorno del espectro autista). Se realizaron diversas actividades y los resultados mostraron efectos muy positivos sobre la motivación y el aprendizaje de los alumnos.

ABSTRACT: The teacher's work means not only a sound knowledge about the students' possible especial needs but also a critical thought and a creative mind for the direct treatment with these students. In the context of the subject "Psychological Foundation for Especial Education" in the degree of Education in the University of La Rioja an innovative experiment was carried out in order to develop the students' competences. In fact, its main objective was to activate the students' critical thought about their previous ideas on the especial educative needs and the knowledge one acquires in the classroom context. Moreover, another objective was to develop their creative mind about the possible activities that a teacher can use with his students, depending on their needs. Two topics were mainly dealt with this methodology: high capacities and disturbance of the autistic spectrum. 
Several activities were carried out and the results proved very positive effects about the students' motivation and learning.

PALABRAS CLAVE: Innovación educativa, pensamiento creativo, pensamiento crítico.

KEYWORDS: Innovative education, creative thought, critical thought.

\section{INTRODUCCIÓN}

La sociedad actual demanda profesionales capaces de gestionar el conocimiento, es decir, capaces de aprender, de usar de distintas formas el saber acumulado y de ser flexibles y críticos en su utilización (Monereo y Pozo, 2003; Pozo y Monereo, 2009). Este planteamiento ha sido especialmente resaltado en el diseño y puesta en marcha de los nuevos planes de estudio siguiendo la filosofía del nuevo Espacio Europeo de Educación Superior. Según este planteamiento, la formación universitaria debe dirigirse a la formación de profesionales competentes, capaces de adaptarse y responder a las demandas cambiantes y flexibles de la sociedad y por tanto, del ejercicio profesional. Entre las competencias que deben desarrollarse se encuentra la de aprender a pensar. Para mejorar nuestra forma de pensar y desarrollar las habilidades de pensamiento es necesario aprender a pensar. Esto se conseguirá mediante el entrenamiento de las habilidades del pensamiento convergente, del pensamiento divergente y de las habilidades metacognitivas (Allueva, 2002; Allueva, 2004).

En este sentido, la universidad española actual se encuentra ante el reto de innovar la manera en la que se enseña y aprende en sus aulas, con el fin de satisfacer la necesidad de hacer a sus alumnos competentes con este fin. Esta necesidad es especialmente patente en estudios como los de Maestro, o actualmente, los nuevos Grados en Educación. La labor de un maestro, entre otras cuestiones, requiere no sólo un conocimiento exhaustivo sobre las posibles necesidades especiales de sus alumnos sino que además debe ser crítico y creativo en las intervenciones que realiza con estos alumnos.

Con el fin de ayudar a desarrollar estas competencias en los alumnos, se llevó a cabo una experiencia cuyos objetivos fueron la activación de un pensamiento crítico sobre las ideas previas que tienen los alumnos acerca de las necesidades educativas especiales y sobre la información que se proporciona en el aula. Asimismo, se pretendía desarrollar un pensamiento creativo sobre posibles actuaciones que pueden realizar con sus propios alumnos ante sus necesidades.

\section{Método}

\section{Participantes}

La experiencia se llevó a cabo en dos grupos de Segundo de la Diplomatura de Maestro de las especialidades de Educación Infantil y de Lengua Extranjera de la Universidad de La Rioja. La asignatura en la que se realizó la experiencia fue la de "Bases psicológicas de la educación especial". Un total de 53 y 43 alumnos estaban matriculados en los dos grupos, respectivamente. 


\section{Objetivos generales perseguidos mediante el desarrollo de las actividades}

- Activación de un pensamiento crítico sobre las ideas previas que tienen los alumnos acerca de las necesidades educativas especiales.

- Desarrollo de un pensamiento crítico de la información que se proporciona en el aula.

- Desarrollo de un pensamiento creativo sobre posibles actuaciones que pueden desarrollar con sus propios alumnos ante sus necesidades.

\section{Planteamiento, procedimiento y resultados de las actividades}

\subsection{Actividades para el tema de altas capacidades}

3.1.1. PRIMERA ACTIVIDAD SOBRE ALTAS CAPACIDADES: ANÁLISIS PERSONAL Y GRUPAL DE MITOS SOBRE ALTAS CAPACIDADES

Objetivos de la primera actividad

- Activación de un pensamiento crítico sobre las ideas previas que tienen los alumnos acerca de las altas capacidades.

- Identificación de ideas erróneas y mitos sobre las altas capacidades.

- Concluir la actividad con un conocimiento científico de la definición de altas capacidades.

- Realización por parte de los alumnos de un análisis metacognitivo pre-post de sus conocimientos.

- Realización por parte de los alumnos de un análisis metacognitivo del proceso de aprendizaje a través del método utilizado.

\section{Procedimiento de la actividad}

Esta actividad se desarrolló el primer día de clase del tema. Antes de comenzar con cualquier explicación sobre el concepto de altas capacidades, se presentaron a los alumnos la siguiente serie de frases sobre las altas capacidades:

- Los alumnos superdotados nacen o se hacen.

- Los alumnos superdotados son los que tienen un Cl mayor de 130.

- ¿Cuándo y desde qué edad se puede diagnosticar la superdotación?

- ¿Hay superdotados en todas las clases sociales?

- Hay más superdotados varones que hembras.

- El superdotado es raro y no tiene vida social.

- Superdotación, talento, genio y altas capacidades son distintos nombres para un mismo fenómeno.

A continuación, se solicitó a los alumnos que analizaran cada una de las afirmaciones presentadas, en primer lugar de forma individual y, posteriormente, en grupos de cuatro o cinco alumnos. Se les requirió que consideraran si estaban de acuerdo o no con dichas afirmaciones y que aportaran información adicional, tanto para apoyar como para refutar las mismas. Para el análisis individual se les dejó diez 
minutos y veinte para el grupal. Pasado el tiempo de discusión, cada grupo eligió a un portavoz y se plantearon en alto las conclusiones a las que se había llegado.

Una por una, se escucharon las argumentaciones y la profesora moderó y corrigió las afirmaciones inadecuadas, resaltando y completando las adecuadas.

\section{Evaluación de la actividad}

La evaluación de esta actividad se realizó a través de dos procedimientos. En primer lugar, se solicitaba a los alumnos que realizaran un análisis metacognitivo del proceso que habían llevado a cabo para contrastar lo aprendido. En segundo lugar, se solicitó a los alumnos que contestaran un cuestionario de evaluación metacognitiva sobre las actividades Ilevadas a cabo para trabajar el tema (Anexo 1).

El análisis metacognitivo del proceso que habían llevado a cabo para contrastar lo aprendido, tenía como objetivo reflexionar sobre lo que pensaban antes de la actividad sobre las altas capacidades y lo que habían aprendido tras la actividad.

La muestra estuvo compuesta por un total de 56 alumnos entre ambos grupos. Los resultados indicaron que un importante número de alumnos mejoraban en la identificación de mitos sobre altas capacidades. Tal y como aparece reflejado en la Tabla 1 y en la Figura 1, un número elevado de alumnos mejoraron en sus respuestas, especialmente en los ítems 2 y 3 que hacían referencia a la importancia relativa del $\mathrm{Cl}$ como criterio diagnóstico en altas capacidades y a la edad adecuada para dicho diagnóstico.

El análisis de los resultados indica, asimismo, que en aquellos ítems en los que la mejora no era tan notoria, los alumnos ya tenían un conocimiento adecuado previo. Este punto fue analizado con los grupos y se constató que una parte importante de los alumnos habían asistido a unas jornadas sobre el tema el curso anterior y por tanto acudían a clase con ideas previas a menudo correctas.

Tabla 1. Número de alumnos/as que mejoran, empeoran o mantienen ideas previas sobre altas capacidades en los diferentes ítems presentados.

\begin{tabular}{|l|c|c|c|c|c|c|c|}
\hline & $\begin{array}{c}\text { Nacen o } \\
\text { se hacen }\end{array}$ & $\mathbf{C I}$ & $\begin{array}{c}\text { Edad } \\
\text { diagnóstico }\end{array}$ & $\begin{array}{c}\text { Clase } \\
\text { social }\end{array}$ & $\begin{array}{c}\text { Más } \\
\text { varones }\end{array}$ & Rareza & $\begin{array}{c}\text { SPD, } \\
\text { talento y } \\
\text { genio }\end{array}$ \\
\hline Mejora & 31 & 39 & 45 & 14 & 29 & 21 & 21 \\
\hline Igual mal & 10 & 26 & 20 & 8 & 9 & 10 & 15 \\
\hline Igual bien & 29 & 5 & 8 & 50 & 33 & 41 & 35 \\
\hline Empeora & 3 & 3 & 0 & 2 & 2 & 1 & 2 \\
\hline
\end{tabular}




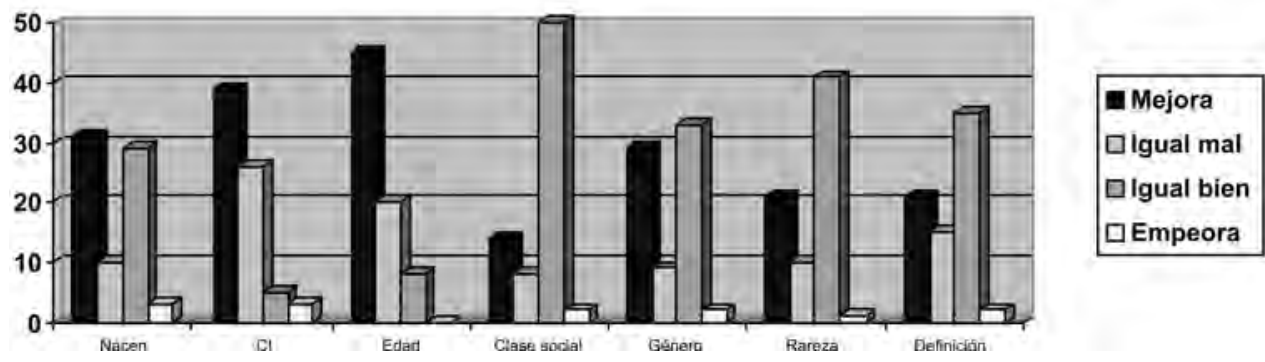

Figura 1. Representación gráfica del rendimiento pre-post de los alumnos tras el trabajo individual y grupal sobre el tema.

\subsubsection{Segunda actividad sobre altas capacidades: documental "Cerebros} asombrosos" del National GeOGraphic

Objetivos de la segunda actividad

- Identificación de ideas erróneas y mitos sobre las altas capacidades.

- Consolidación del conocimiento adquirido sobre la definición de altas capacidades.

\section{Procedimiento de la actividad}

Esta actividad se desarrolló el tercer día de clase del tema. Una vez realizada la primera actividad y visto el apartado de definición de altas capacidades, los alumnos vieron el documental propuesto. Debían coger notas sobre:

- Ideas apropiadas (contrastadas y basadas en la ciencia) sobre las altas capacidades.

- Ideas erróneas que contiene el documental.

A continuación, se solicitó a los alumnos que entregaran sus conclusiones a la profesora y que, además, las discutieran con todo el grupo. La profesora actuó de moderadora del proceso.

\section{Evaluación de la actividad}

La evaluación de esta actividad se realizó preguntando directamente a los alumnos hasta qué punto consideraban que había sido efectiva para la consecución de los objetivos. Asimismo, contestaron el cuestionario de evaluación metacognitiva de la actividad al final del tema.

Los alumnos realizaron dicho trabajo de manera individual. Dada la imposibilidad de realizar un análisis cualitativo de cada uno de los documentos entregados, se llevó a cabo una reflexión por parte de las docentes de la que resultaron las siguientes conclusiones: 
- Importancia de la implicación del entorno (familia y sistema educativo fundamentalmente) para la cristalización de las altas capacidades, incluso en el periodo gestacional.

- De acuerdo con Renzulli (1994), importancia de la motivación y de la implicación en la tarea en el desarrollo de la alta capacidad.

- Necesidad de potencial a nivel genético de la alta capacidad.

- Existencia de periodos críticos para el desarrollo de conexiones neuronales.

\subsubsection{TERCERA ACTIVIDAD SOBRE ALTAS CAPACIDADES: PROPUESTA DE ACTIVIDADES DE ENRIQUECIMIENTO EN EL AULA O EXTRACURRICULARES PARA LOS ALUMNOS CON ALTAS CAPACIDADES}

Objetivos de la tercera actividad

- Desarrollo un pensamiento creativo sobre posibles actuaciones que pueden desarrollar con sus propios alumnos ante sus necesidades.

\section{Procedimiento de la actividad}

Esta actividad se desarrolló el cuarto día de clase del tema. La sesión se dedicó a analizar las diferentes respuestas educativas que pueden ofrecerse a los alumnos con altas capacidades, dentro y fuera del contexto escolar.

Una vez presentadas las diferentes opciones posibles, los alumnos se reunieron en grupos de $4 / 5$ personas y diseñaron una actividad de enriquecimiento para un supuesto alumno o grupo de alumnos con altas capacidades.

El procedimiento en grupo fue el siguiente:

- Utilización de la técnica Brainstorming para seleccionar posibles actividades.

- Utilización de los pasos del proceso creativo para la propuesta inicial de una actividad de enriquecimiento:

- Presentación

- Preparación

- Generación de respuestas

- Validación

- Aplicación y toma de decisiones

- Entrega a la profesora y presentación al resto de los compañeros.

\section{Evaluación de la actividad}

La clase dedicó un tiempo para exponer y realizar las actividades propuestas por los alumnos (en concreto, se llevaron a cabo 6 actividades). El objetivo fue comprobar que son útiles para lo que se pretende, al tiempo que ayuda a desarrollar la creatividad en los propios compañeros. Los informes grupales fueron recogidos para ser evaluados por las profesoras y formar parte de la nota de prácticas de la asignatura. 
Evaluación metacognitiva de las tres actividades llevadas a cabo en el tema de las altas capacidades.

El objetivo de esta evaluación era analizar si las actividades llevadas a cabo para el estudio del tema, eran consideradas adecuadas por parte de los alumnos, en el sentido de que percibían estas actividades como enriquecedoras de su aprendizaje. En concreto se presentó a los alumnos el cuestionario que se adjunta como Anexo 1.

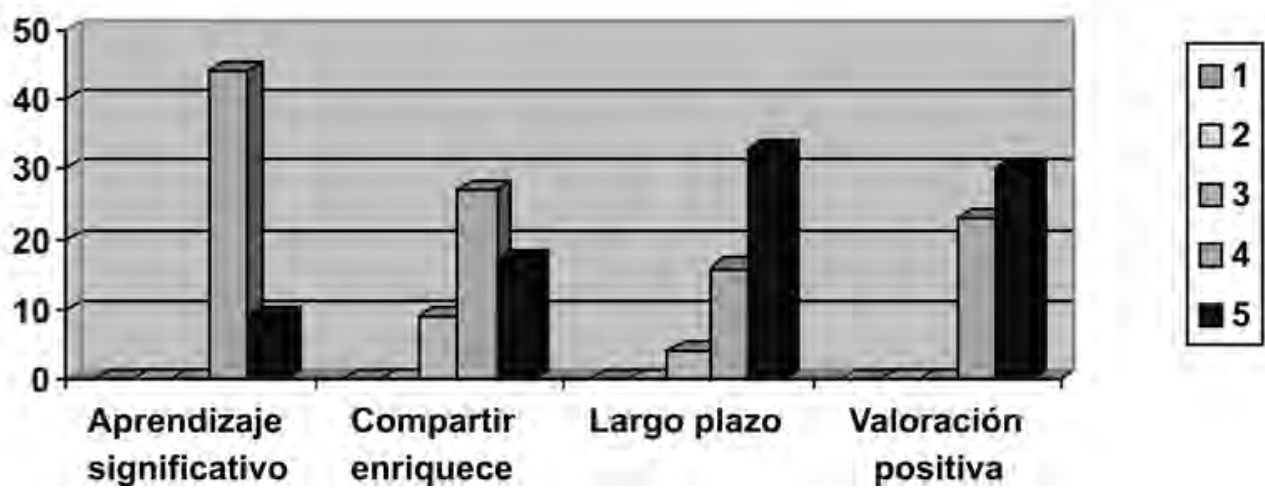

Figura 2. Análisis metacognitivo por parte de los alumnos.

Los resultados del análisis de las respuestas por parte de los alumnos mostraron un panorama muy positivo. Tal y como puede apreciarse en la Figura 2, los alumnos afirmaron que las actividades sobre el tema de altas capacidades les habían permitido alcanzar un aprendizaje más significativo que las estrategias más receptivas (media $=4.16$ sobre 5 puntos). Asimismo, los alumnos afirmaron que el hecho de incluir actividades de discusión en grupo y generales moderadas por las docentes enriquecía su aprendizaje (media $=4.15$ sobre 5 puntos).

Por otra parte, los alumnos afirmaron que estaban totalmente de acuerdo en que, aunque este tipo de actividades conlleva más tiempo, recordarían a más largo plazo lo aprendido (media $=4.54$ sobre 5 puntos). Por último, la valoración general de las tres actividades fue altamente positiva (media $=4.56$ sobre 5 puntos).

\subsection{Actividades para el tema del trastorno del espectro autista}

3.2.1. Primera aCtIVIDAD SOBRE EL TRASTORNO DEL ESPECTRO AUTISTA: ANÁLISIS PERSONAL Y GRUPAL DE IDEAS ERRÓNEAS SOBRE PERSONAS CON TRASTORNO DE ESPECTRO AUTISTA Y ANÁLISIS METACOGNITIVO SOBRE LA PROPIA CAPACIDAD PARA AFRONTAR LA TAREA PROPUESTA

Objetivos de la primera actividad

- Activación de un pensamiento crítico sobre las ideas previas que tienen los alumnos acerca del trastorno del espectro autista. 
- Concluir la actividad con un conocimiento científico de la definición del trastorno del espectro autista.

- Realización por parte de los alumnos de un análisis metacognitivo pre-post de sus conocimientos.

\section{Procedimiento de la actividad}

Esta actividad se desarrolló el primer día de clase del tema. Antes de comenzar con cualquier explicación sobre el concepto del trastorno de espectro autista, se solicitó a los alumnos que realizaran un juicio metacogntivo (Fase $I$ ) previo a la discusión sobre ideas erróneas (el cuestionario se presenta en el Anexo 2 al presente documento).

A continuación, se procedió a presentar a los alumnos la siguiente serie de frases sobre el trastorno del espectro autista:

- El autismo es una forma inicial de esquizofrenia

- La mayor parte de los niños tienen una inteligencia normal

- Actualmente se considera que el autismo es básicamente un desequilibrio emocional.

- Los niños autistas viven aislados en su mundo de fantasía.

- Los autistas tienen un comportamiento semejante a los sordos.

Posteriormente (Fase 2) se solicitó a los alumnos que analizaran en primer lugar individualmente y posteriormente por grupos de cuatro o cinco alumnos cada una de las afirmaciones presentadas en la transparencia. Se les requirió que examinaran si estaban de acuerdo o no con dichas afirmaciones y que aportaran información adicional tanto para apoyar como para refutar las mismas. Para el análisis individual se les dejó diez minutos y para el grupal veinte.

Pasado el tiempo de discusión, cada grupo eligió a un portavoz y se plantearon en alto las conclusiones a las que había llegado.

Una por una, se escucharon las argumentaciones, y la profesora moderó y corrigió las afirmaciones inadecuadas, resaltando y completando las adecuadas.

Finalmente, se procedió a realizar la última fase del juicio metacognitivo (Fase 3, Anexo 2) sobre la percepción que los alumnos tenían acerca de su capacidad para enfrentar tareas de este tipo.

\section{Evaluación de la actividad}

La muestra estuvo compuesta por un total de 45 alumnos entre ambos grupos. Los resultados indicaron que la mayoría de alumnos mejoraban en la identificación de ideas erróneas sobre el trastorno del espectro autista. Tal y como aparece reflejado en la Tabla 2 y en la Figura 3, un número muy elevado de alumnos mejoraron en sus respuestas. Los únicos ítems que no mostraron resultados tan sorprendentes fueron los que planteaban la semejanza entre el trastorno del espectro autista y la esquizofrenia y la deficiencia auditiva. En estos casos, los alumnos fueron capaces de identificar y justificar desde un inicio la inadecuación de la afirmación. 
Tabla 2. Número de alumnos/as que mejoran, empeoran o mantienen ideas previas sobre el trastorno del espectro autista en los diferentes ítems presentados.

\begin{tabular}{|l|c|c|c|c|c|}
\hline & Esquizofrenia & Inteligencia & Desequilibrio & Fantasía & Sordos \\
\hline Mejora & 27 & 45 & 39 & 39 & 27 \\
\hline Igual mal & 0 & 0 & 0 & 3 & 3 \\
\hline Igual bien & 18 & 0 & 6 & 3 & 12 \\
\hline Empeora & 0 & 0 & 0 & 0 & 0 \\
\hline
\end{tabular}

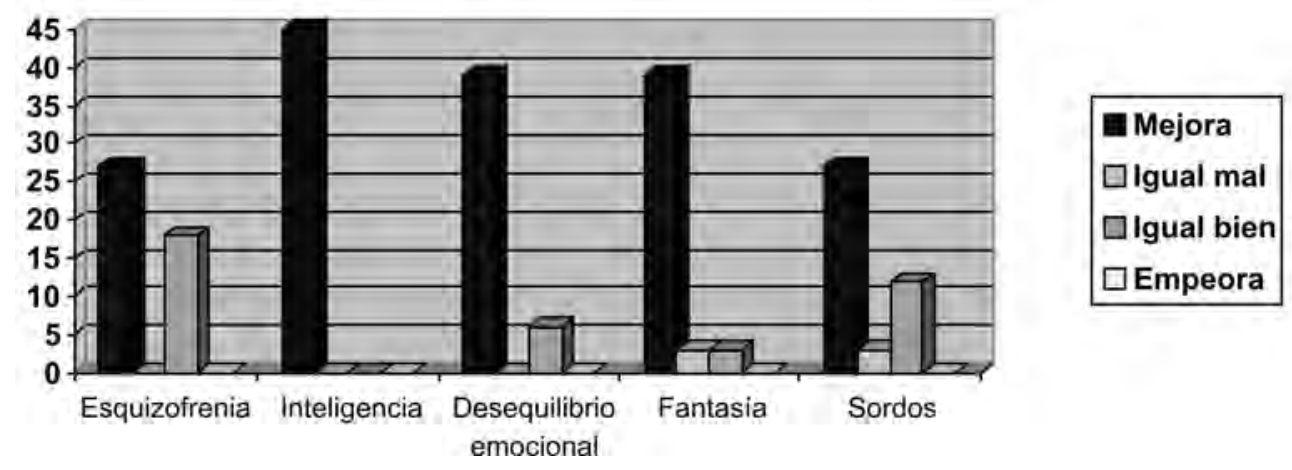

Figura 3. Representación gráfica del rendimiento pre-post de los alumnos tras el trabajo individual y grupal sobre el tema.

Respecto al juicio metacognitivo acerca de la capacidad percibida para realizar este tipo de tareas, los resultados fueron muy positivos.

Del total de alumnos implicados en las clases, se consiguió una respuesta completa de ambas fases de 45 alumnos. Los resultados mostraron una evolución positiva sobre la evaluación metacognitiva que realizan los alumnos de sus propios conocimientos y capacidad para hacer frente a la tarea propuesta.

Como puede observarse en la Figura 4, antes de realizar las actividades (Fase 1) los alumnos afirmaron no sentirse capaces de contestar a las preguntas sobre autismo en un 20\% de los casos; el $66.7 \%$ no sabía si sería capaz; sólo un $13.3 \%$ afirmaron estar preparados. Tras la exposición del tema (Fase 3), el 86.7\% afirmaban sentirse capaces de contestar a dichas preguntas. Sólo el 6.7\% afirmaron no verse capaces, y el $6.7 \%$ no sabían si serían capaces. 


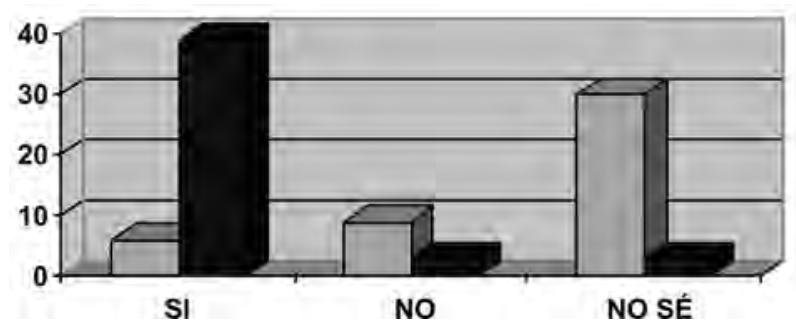

Figura 4. Juicio Metacognitivo sobre capacidad percibida.

Las respuestas sobre la percepción que tenían los alumnos sobre sus conocimientos muestran unos resultados algo diferentes. En la Figura 5 se observa cómo muchos alumnos cambian la percepción sobre sus conocimientos hacia mayor seguridad (del $6.7 \%$ al $53.3 \%$ ). No obstante, son muchos los alumnos (60\% antes de las actividades y $33.3 \%$ después) que consideran que sus conocimientos no son suficientes o que no saben si poseen los conocimientos necesarios $(33.3 \%$ antes y $13.3 \%$ después). Por tanto, aunque la mayor parte de los alumnos mejoran la percepción tras las actividades, se constata que alrededor del $45 \%$ de alumnos afirma que continúa sintiéndose inseguros. Este resultado no debe ser interpretado de manera negativa ya que a menudo nuestro objetivo como docentes consiste precisamente en hacer conscientes a los alumnos de la necesidad de mayor estudio y preparación para poder intervenir con los casos en el aula.

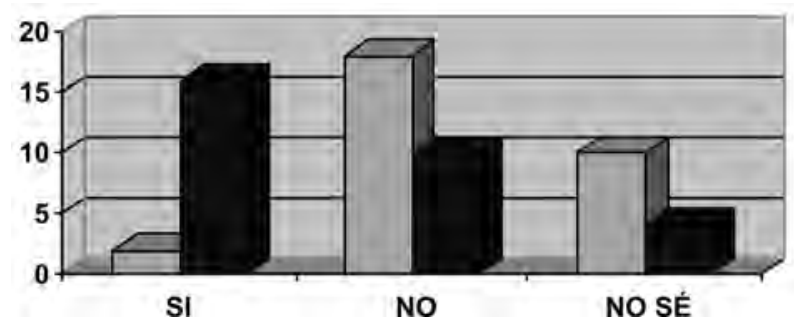

\section{$\square$ Antes de las actividades \\ Después de las actividades}

Figura 5. Juicio Metacognitivo sobre conocimientos necesarios.

\subsubsection{SeGunda ACtIVIDAD SOBRE EL tRASTORNO DEL ESPECTRO AUtista: CONFERENCIA POR PARTE DE UN EXPERTO EN EL TRASTORNO DEL ESPECTRO AUTISTA}

\section{Objetivos y procedimiento de la actividad}

Con el objetivo de que los alumnos conocieran con precisión los recursos con los que se cuenta en la Comunidad de La Rioja para niños con este trastorno, las docentes se pusieron en contacto con la Asociación ARPA (Asociación Riojana de Personas con Autismo). Esta asociación proporcionó el contacto con el psicólogo de la misma, persona encargada de la formación en centros educativos en los que están integrados los niños con este trastorno. 
El psicólogo estuvo dispuesto a colaborar con el Departamento de Ciencias de la Educación y ofreció una excelente conferencia en la que se resaltaron los aspectos fundamentales para la intervención con estos niños. Los alumnos valoraron muy positivamente la actividad.

\section{CONCLUSIONES}

El presente trabajo tenía como objetivos: a) la activación de un pensamiento crítico sobre las ideas previas que tienen los alumnos acerca de las necesidades educativas especiales, b) el desarrollo de un pensamiento crítico de la información que se proporciona en el aula y c) desarrollo de un pensamiento creativo sobre posibles actuaciones que pueden desarrollar con sus propios alumnos ante sus necesidades.

Para la consecución de dichos objetivos se seleccionaron dos de los temas del programa: altas capacidades y trastorno del espectro autista. Se realizaron diversas actividades y los resultados mostraron efectos muy positivos sobre la motivación y aprendizaje de los alumnos.

En primer lugar, las tres actividades planteadas para el tema de altas capacidades mostraron que los alumnos, una vez realizadas, eran capaces de reconocer su falta de conocimiento inicial y su grado de aprendizaje posterior. Asimismo, afirmaron estar más motivados e implicados en el estudio de este tema. Por otra parte, los resultados del análisis metacognitivo mostraron que percibían su aprendizaje como más significativo, se habían enriquecido con las actividades grupales, pensaban que recordarían el tema a mayor largo plazo y valoraban muy positivamente la manera en la que se había trabajado el tema.

En segundo lugar, para las actividades sobre el trastorno de espectro autista, los alumnos mostraron una mejora significativa de sus conocimientos. De igual manera, los resultados sobre el juicio metacognitivo acerca de la capacidad percibida para realizar este tipo de tareas, fueron muy positivos. Un dato que debe resaltarse es en relación a la percepción que tenían los alumnos sobre sus conocimientos tras trabajar el tema. Un número importante de alumnos seguía sintiendo inseguridad a la hora de resolver tareas relacionadas con el trastorno del espectro autista. La discusión de este resultado con los alumnos puso de manifiesto que los alumnos eran conscientes de la necesidad de mayor estudio y preparación para poder intervenir con los casos en el aula.

En conjunto, los resultados se valoran de manera muy positiva tanto por parte de los alumnos como de las docentes. No obstante, es importante resaltar que este tipo de actividades conlleva una gran inversión por parte del docente, especialmente las fases de evaluación de las actividades. Por otra parte, es también necesario reconocer que este tipo de actividades no pueden llevarse a cabo sin la implicación por parte de los estudiantes. Las tareas propuestas conllevan mucho tiempo de discusión y trabajo en el aula y no pueden llevarse a cabo sin ser acompañadas por el estudio por parte del alumno fuera del aula. 


\section{REFERENCIAS BIBLIOGRÁFICAS}

ALLUEVA, P. (2002). Desarrollo de habilidades metacognitivas: Programa de intervención. Zaragoza: Consejería de Educación y Ciencia. Diputación General de Aragón.

ALLUEVA, P. (2004). Desarrollo del pensamiento creativo en el ámbito universitario. Anuario de Filosofía y Psicología, 7, 117-130.

MONEREO, C. y POZO, J. I. (2003). La cultura educativa en la Universidad: Nuevos retos para profesores y alumnos. En C. Monereo y J. I. Pozo (Eds.), La Universidad ante la nueva cultura educativa. Enseñar y aprender para la autonomía (pp. 15-29). Madrid: Síntesis.

POZO, J. I. y MONEREO, J. I. (2009). Introducción: La nueva cultura del aprendizaje universitario o por qué cambiar nuestras formas de enseñar y aprender. En J. I. Pozo y M. P. Pérez Echeverría (Coors.), Psicología del aprendizaje universitario: La formación en competencias (pp. 9-28). Madrid: Morata.

RENZULLI, J. (1994). El concepto de los tres anillos de la superdotación: Un modelo de desarrollo para una producción creativa. En Benito, Y. (coord.), Desarrollo y educación de los niños superdotados. Salamanca: Amarú. 


\section{Anexo 1. Cuestionario utilizado para la evaluación metacognitiva REALIZADA SOBRE LAS ACTIVIDADES EN EL TEMA DE ALTAS CAPACIDADES}

\begin{tabular}{|l|l|l|l|l|l|}
\hline \multicolumn{5}{|c|}{ Evaluación metacognitiva de la actividad } \\
\hline $\begin{array}{l}\text { A continuación se presentan una serie de afirmaciones sobre la forma en la que } \\
\text { has estudiado el tema. Por favor, señala la opción que mejor describa tu postura: }\end{array}$ \\
\hline Esta forma de estudiar el tema: & $\begin{array}{l}\text { Totalmente } \\
\text { de acuerdo }\end{array}$ & $\begin{array}{l}\text { De } \\
\text { acuerdo }\end{array}$ & $\begin{array}{l}\text { Ni de } \\
\text { acuerdo ni } \\
\text { en desacuerdo }\end{array}$ & $\begin{array}{l}\text { En desacuerdo } \\
\text { en desacuerdo }\end{array}$ & $\begin{array}{l}\text { Totalmente } \\
\text { en }\end{array}$ \\
\hline $\begin{array}{l}\text { 1. Me ha permitido alcanzar } \\
\text { un aprendizaje más signi- } \\
\text { ficativo que otro tipo de } \\
\text { estrategias más receptivas. }\end{array}$ & & & & \\
\hline $\begin{array}{l}\text { 2. Me ha permitido consta- } \\
\text { tar que compartir mis pro- } \\
\text { puestas con otras personas } \\
\text { enrique mi aprendizaje. }\end{array}$ & & & & & \\
\hline $\begin{array}{l}\text { 3. Aunque conlleva más } \\
\text { tiempo, creo que recor- } \\
\text { daré a largo plazo los } \\
\text { contenidos aprendidos. }\end{array}$ & & & & & \\
\hline
\end{tabular}




\section{ANeXo 2. Cuestionario iniCial para la actiVidad sobre el trastorno DEL ESPECTRO AUTISTA}

\section{Fase I}

Contesta las siguientes preguntas, ordenadamente, una después de otra.

1. ¿Crees que serás capaz de contestar algunas preguntas sobre el autismo?
$\square$ Creo que sí
$\square$ Creo que no
$\square$ No lo sé

2. ¿Crees que tienes conocimientos necesarios para resolver ejercicios de este tipo?
$\square$ Creo que sí
$\square$ Creo que no
$\square$ No lo sé

2. ¿Qué conocimientos crees que te faltan para este fin?

\section{Fase 2}

Contesta las siguientes preguntas, ordenadamente, una después de otra.

1. ¿Crees que has sido capaz de de contestar algunas preguntas sobre el autismo?

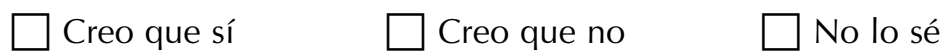

2. ¿Crees que tenías los conocimientos necesarios para resolver el ejercicio?

$\square$ Creo que sí $\quad \square$ Creo que no $\quad \square$ No lo sé

3. ¿Qué conocimientos crees que te faltan para resolver ejercicios de este tipo? 